\title{
Image Processing in the Optical Microscopy: Decoding, Photometry Correction and Compression
}

\author{
http://dx.doi.org/10.3991/ijes.v2i2.3699 \\ Waheb LARBI, Allal LARBI, Bachir DJEDOU \\ Université Badji Mokhtar, Annaba, Algeria
}

\begin{abstract}
This article presents an application of the digital imaging in the reading of a slide in optical microscopy, by applying a photometric correction of images issued out of the microscope, an interpolation of images captured by a CDD sensor (decoding) and the implementation of a mosaicking in order to obtain a virtual slide of big size. This study is particularly decisive in a context of telediagnosis applications in optical microscopy, using this type of sensor and manipulating images of large dimensions. We also propose some solutions which improve the efficiency of the telediagnosis chain, and the quality of the visualized images.
\end{abstract}

Index Terms-image processing, decoding, optical microscopy, interpolation, compression.

\section{INTERPOLATION AND DECODING}

The microscope is equipped with a CCD sensor based camera. To acquire a coloured image, these sensors use a network of filters, introduced by BAYER in 1976 [1] [2].

The pixels partition of the camera issued image is given at figure 9.

This format is called 'BAYER' (see table 1).

As we can see, the green channel is sampled on the half of the total number of the image pixels, but every one of the blue and the red channels occupies the quarter of the number of pixels. We denote this format by Y8.

TABLE I.

THE PIXELS PARTITION IN ‘BAYER’ FORMAT.

\begin{tabular}{|l|l|l|l|l|l|l|l|}
\hline G & B & G & B & G & B & G & B \\
\hline R & G & R & G & R & G & R & G \\
\hline G & B & G & B & G & B & G & B \\
\hline R & G & R & G & R & G & R & G \\
\hline G & B & G & B & G & B & G & B \\
\hline R & G & R & G & R & G & R & G \\
\hline G & B & G & B & G & B & G & B \\
\hline R & G & R & G & R & G & R & G \\
\hline G & B & G & B & G & B & G & B \\
\hline R & G & R & G & R & G & R & G \\
\hline
\end{tabular}

In order to go from 'BAYER' format to 'RGB' format, the camera makes an interpolation to retrieve the two missing colours in every image pixel, (See Fig. 1 and Fig $3)$.

This problem is the object of several recent publications [3] [4].
To improve the efficiency of the telediagnosis chain, we will use the adjusted interpolation method of correlation of the linear interpolation version proposed in [5], which is fast and easy to implement (see table 2 and table $3)$.

The two colours R and B are linearly interpolated with the nearest neighbours of the same colour.

There are four possible cases:

- When we are in a green pixel 'G' (See table II), two cases are discussed:

- Interpolate the two missing colours ' $R$ ' and ' $B$ ',

$\circ$ Take the average of the two nearest neighbours of the same colour.

- When we are in an ' $\mathrm{R}$ ' or 'B' pixel (See table III), in these two cases we take the average of the four nearest neighbours around the ' $\mathrm{R}$ ' or ' $\mathrm{B}$ ' pixel.

TABLE II.

INTERPOLATION OF THE TWO MISSING COLOURS WHEN WE ARE IN GREEN PIXEL

\begin{tabular}{|l|l|l|}
\hline $\mathbf{G}$ & $\mathbf{B}_{1}$ & $\mathbf{G}$ \\
\hline $\mathbf{R}_{1}$ & $\mathrm{G}$ & $\mathbf{R}_{\mathbf{2}}$ \\
\hline $\mathbf{G}$ & $\mathbf{B}_{2}$ & $\mathbf{G}$ \\
\hline
\end{tabular}

\begin{tabular}{|l|l|l|}
\hline $\mathbf{G}_{1}$ & $\mathbf{R}_{\mathbf{1}}$ & $\mathbf{G}_{\mathbf{2}}$ \\
\hline $\mathbf{B}_{1}$ & $\mathrm{G}$ & $\mathbf{B}_{\mathbf{2}}$ \\
\hline $\mathbf{G}_{3}$ & $\mathbf{R}_{\mathbf{2}}$ & $\mathbf{G}_{\mathbf{4}}$ \\
\hline
\end{tabular}

$\mathbf{R}=\left(\mathbf{R}_{1}+\mathbf{R}_{2}\right) / 2$

$B=\left(B_{1}+B_{2}\right) / 2$

TABLE III

INTERPOLATION OF THE TWO COLOURS R AND B WHEN WE ARE IN RED OR BLUE PIXEL

\begin{tabular}{|l|l|l|}
\hline $\mathbf{B}_{1}$ & $\mathbf{G}_{1}$ & $\mathbf{B}_{2}$ \\
\hline $\mathbf{G}_{4}$ & $\mathbf{R}$ & $\mathbf{G}_{\mathbf{2}}$ \\
\hline $\mathbf{B}_{3}$ & $\mathbf{G}_{3}$ & $\mathbf{B}_{\mathbf{4}}$ \\
\hline
\end{tabular}$\quad$\begin{tabular}{|l|l|l|}
\hline $\mathbf{R}_{\mathbf{1}}$ & $\mathbf{G}_{1}$ & $\mathbf{R}_{\mathbf{2}}$ \\
\hline $\mathbf{G}_{\mathbf{4}}$ & $\mathrm{B}_{\mathbf{3}}$ & $\mathbf{G}_{\mathbf{2}}$ \\
\hline $\mathbf{R}_{\mathbf{3}}$ & $\mathbf{G}_{3}$ & $\mathbf{R}_{\mathbf{4}}$ \\
\hline
\end{tabular}

$$
\begin{aligned}
& R=\left(R_{1}+R_{2}+R_{3}+R_{4}\right) / 4 \\
& B=\left(B_{1}+B_{2}+B_{3}+B_{4}\right) / 4
\end{aligned}
$$


(1)

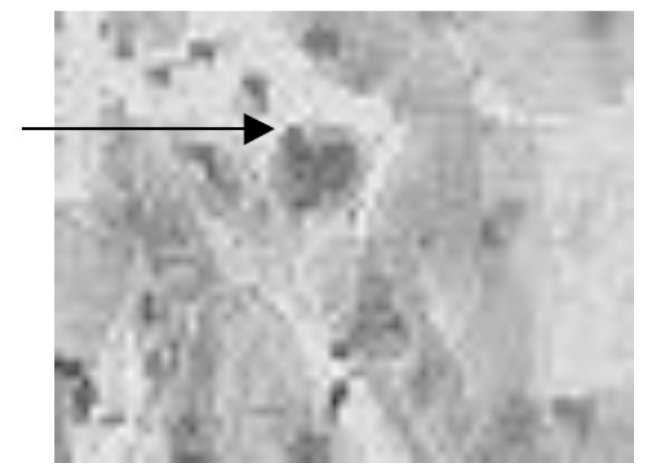

Figure 1. Blue component interpolated by the camera software (zoom 2X)

(1) Crystallization effect.

(2)

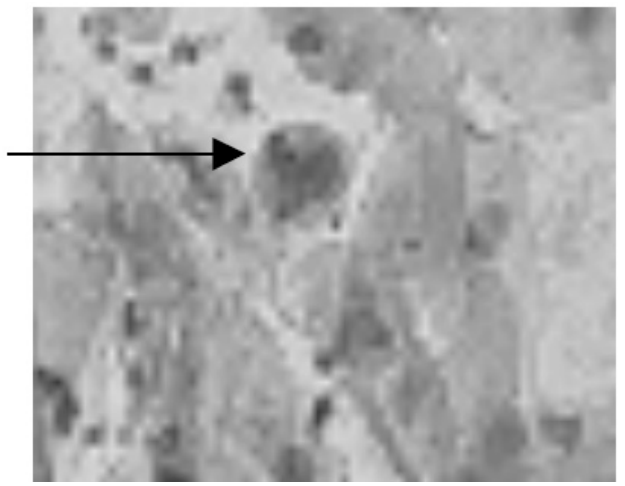

Figure 2. Blue component interpolated by the developed software (zoom 2X)

(2) Correction of the crystallization default.

(3)

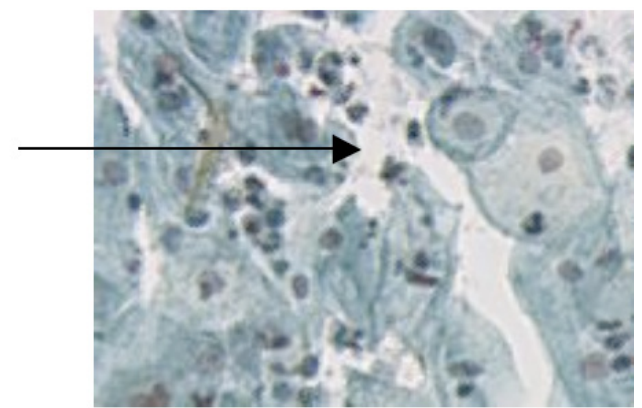

Figure 3. Y8 image interpolated by the camera software (zoom 2X)

(3) Presence of redness.

(4)

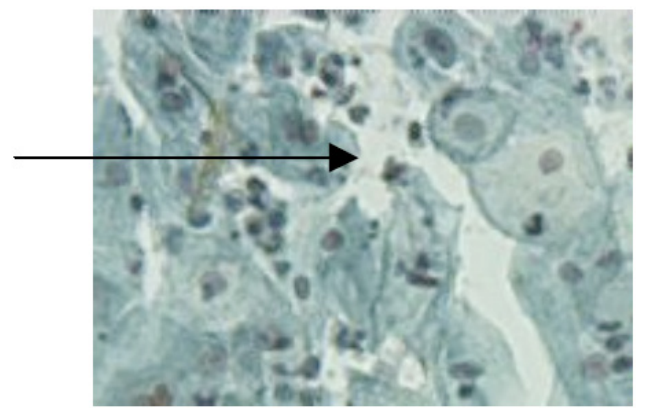

Figure 4. Y8 image interpolated by the developed software (zoom 2X)

(4) Disappearance of the redness.
II. LARGE SLIDE AND PHOTOMETRY DEFAULT:

The observation of the large field image, obtained by the mosaicking process which is used in the telediagnosis chain, shows a grid effect visible at the levels of join borders of the microscope issued images (see Fig.5). This effect is due to the inhomogeneous illumination of the slide scene.
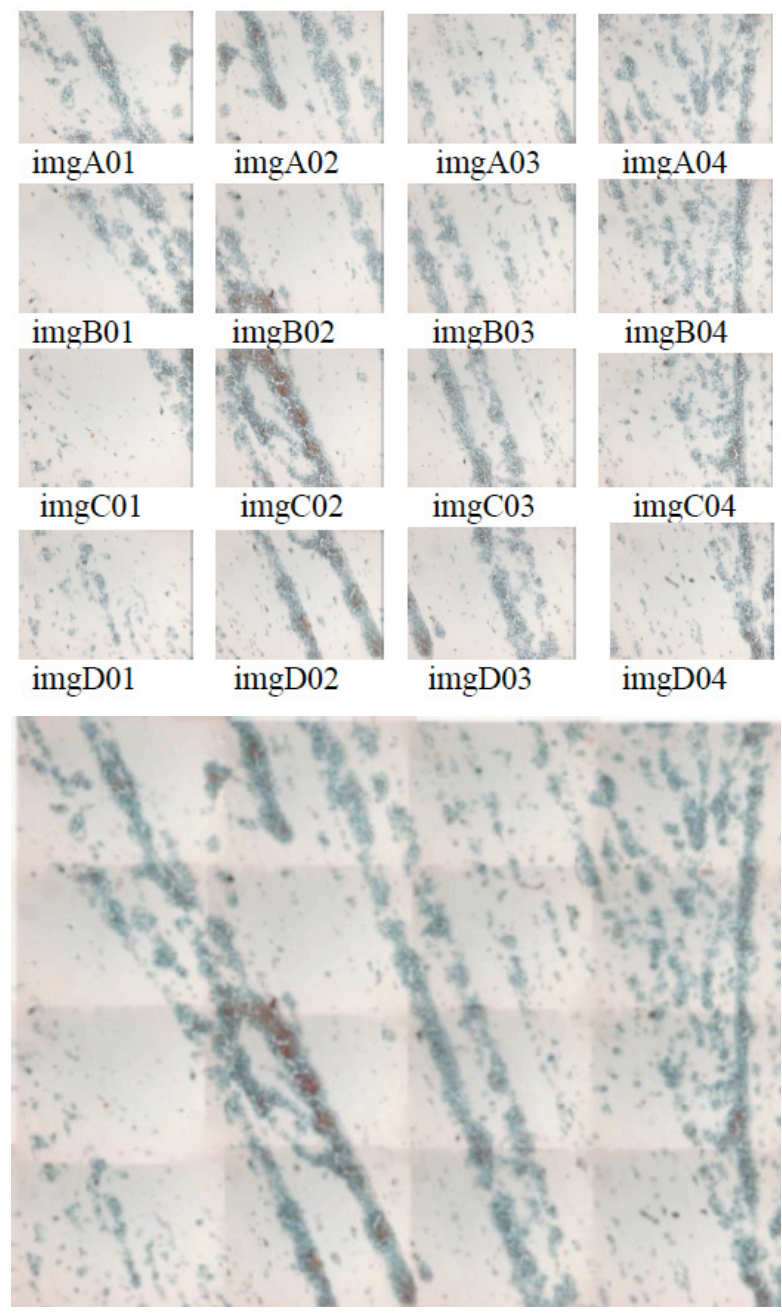

Figure 5. The set of images to mosaick and result of the mosaicking before the photometry correction

An histogram stretching of the image allows to show this default.

This technique consists on applying a linear transformation on the image of empty scene (See Fig. 6). This transformation is called " histogram stretching " (see Fig.6 and Fig. 7).

Let $g_{\min }$ and $g_{\max }$ be the minimum and the maximum grey levels of the image pixels. The stretching consists on the application of a linear function $f$ to the image grey levels, such that :

$$
f\left(g_{\text {min }}\right)=0 \text { and } f\left(g_{\text {max }}\right)=255 .
$$

We have then :

$$
f(x)=255\left(x-g_{\min }\right) /\left(g_{\max }-g_{\min }\right)
$$



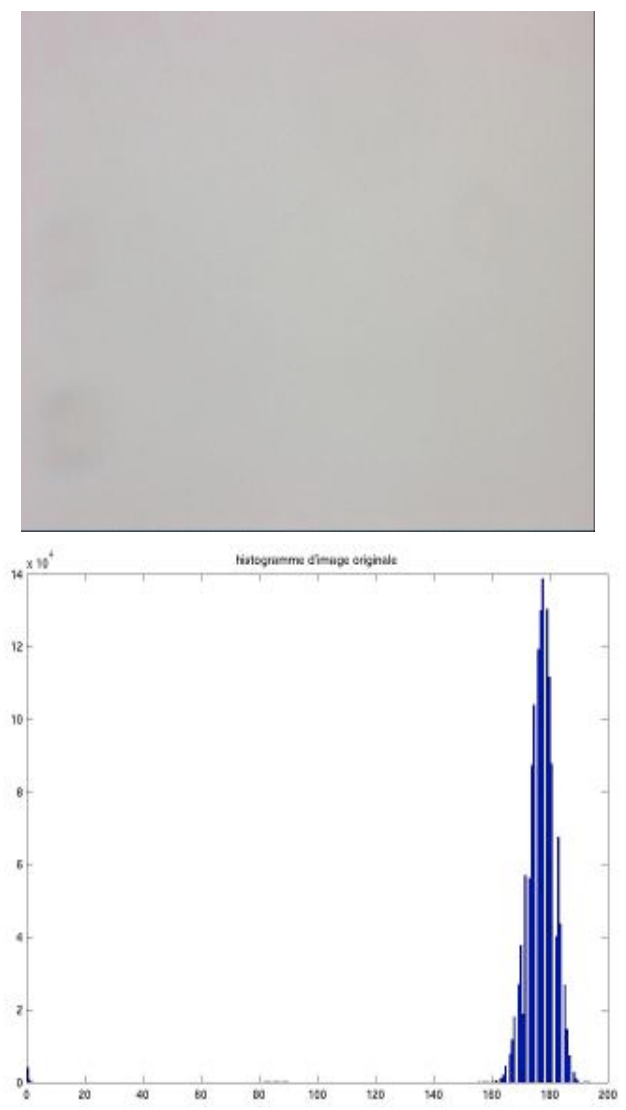

Figure 6. Initial image of the 'shading' empty scene and histogram of the original image of the shading
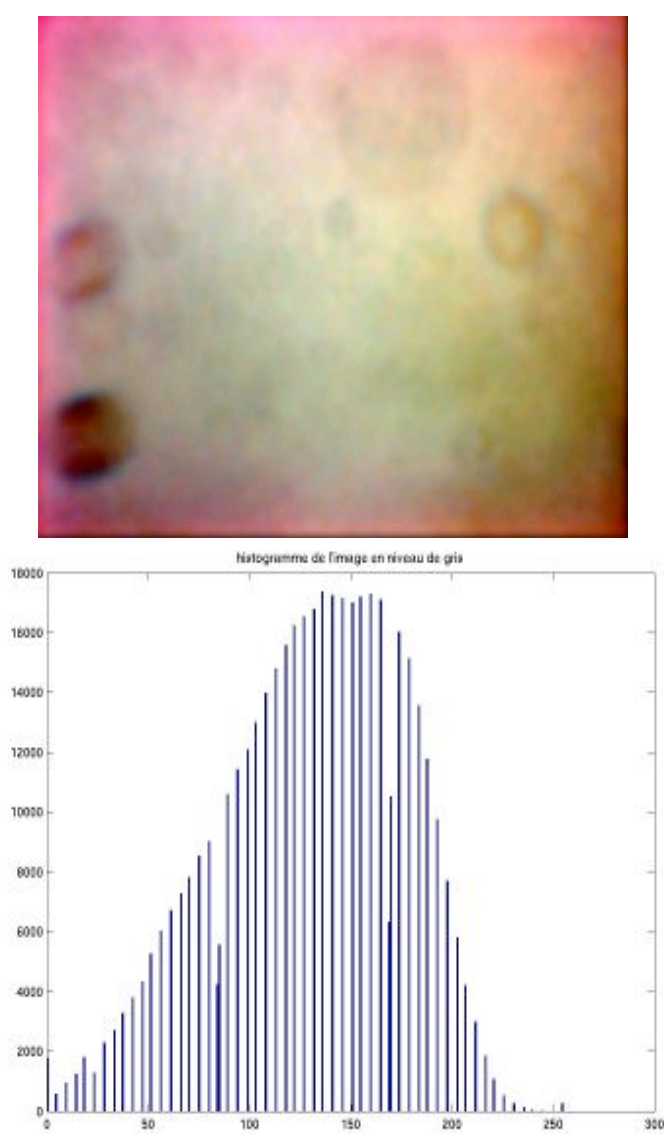

Figure 7. Image of shading after histogram stretching and histogram after histogram stretching
The illumination default, well visible now (see Fig. 7), introduces the grid effect on the final mosaicked image.

\section{PHOTOMETRY CORRECTION:}

To correct this default, we propose:

- The application of an approximation of the illumination function on each image, each image to mosaick will be divided by this function (see Fig.7).

- The point by point correction. (Divide each image on its shading image).

Nevertheless, the scene black marks which represent the dust on the camera objective are complex to model analytically. Then we have chosen to make a point per point correction:
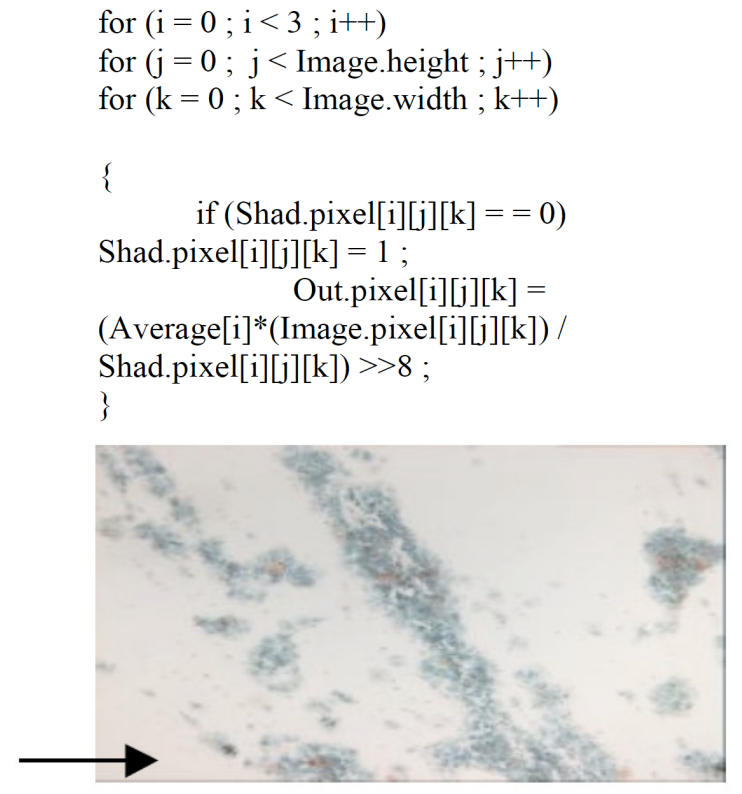

(6)

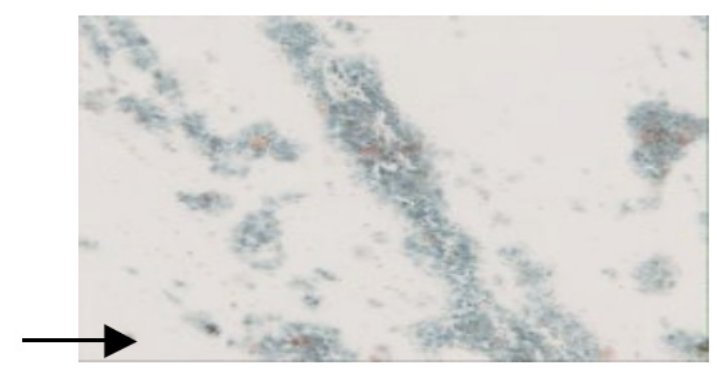

Figure 8. Original image to mosaic before and after shading correction

(5) A redness appears at the image borders due to non homogenous illumination of the slide scene

(6) Disappearance of the redness after photometry correction

The shading correction can be applied on the camera issued images, this reduces the execution time of three times (division over one channel instead of three channels for colour images). Then, a decoding will be applied on the correction results in order to obtain the colour images.

\section{IMAGES MOSAICKING:}

The images obtained by microscopy are naturally very local. It is though necessary to replace them in a more global context, which is generally done using a diminution of the magnification. 
The proposed project suggests to develop an alternative method which consists, on the contrary, to take several shifted images at a unique resolution. In order to obtain one image, you have to align all these images the most finely possible. Of course, this alignment has to be done automatically.

\section{A. Determination of the joins borders :}

After the photometric correction, the elementary images are readjusted:

For two images we look for the superposition and the correspondence of each pixel of the recovering zone.

To obtain an image of large field, the fact to piece elementary images together creates artefacts due to the apparition of an artificial border at the level of the joins. That's why we are looking for an optimal join border, following certain criteria:

- The join border must follow the common edges of the two images, so that the possible visible transition already exists and therefore be natural.

- We must not create new contours in the mosaic.

- The residual geometric disparity must be minimal.

The correlation method is used to put in correspondence pixels in a recovering zone. The idea is to define a similarity measurement between the pixels of two images [6].

Looking for the correspondence between two images $f$ and $g$ is looking for maximum of the correlation coefficient $\left|\mathrm{C}_{f g}\right|$.

We obtain, then, a sequence of correlation values, and the pixel corresponding to the best score will be chosen as matching the pixel of the centre of the fixed window in the other image [7].

The correspondence per correlation is a very efficient method in the case where the couple of images presents regions allowing to ease the matching [8][9].

\section{B. Mosaicking of two images :}

The microscope issued images whose recovering zone is $85 \%$ wide, meaning a recovering zone of $15 \%$ (see Fig.9)

Making the mosaicking using the mosaicking package 'Panavue' [10][11] (see Fig. 10a ), then using the developed program (see Fig.10b), with a binary weighing of $(0,1)$ and $(1,0)$ of the photometry in the recovering zone, and finally a progressive weighing from 0 to 1 always in the recovering zone, we note that the mosaicking time of two images by 'PANAVUE' takes $39 \mathrm{~s}$ whereas the mosaicking by the developed program is about only $13 \mathrm{~s}$.
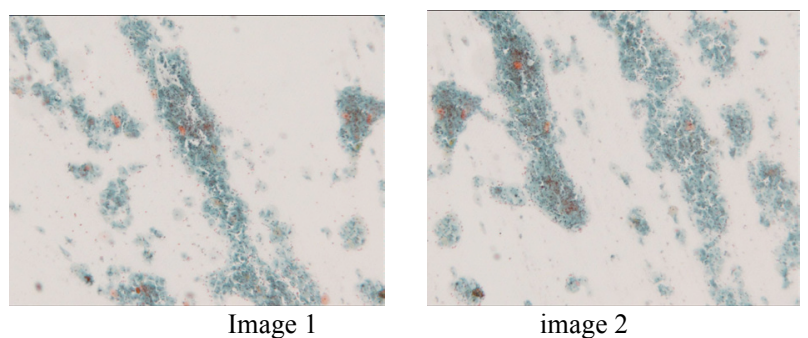

Figure 9. Image 1 and Image 2 to mosaick

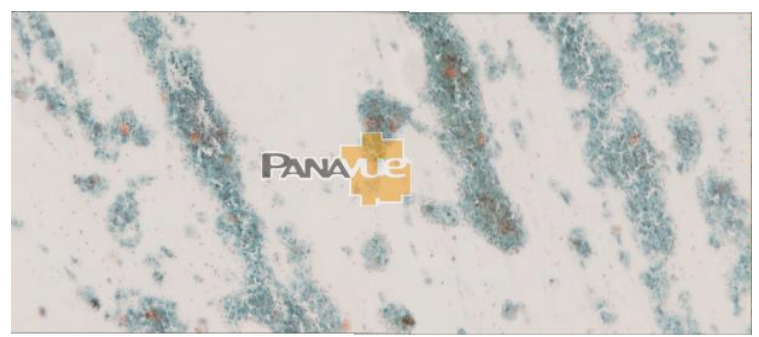

a) Mosaicking result of the two images 'Fig.9' by the 'Panavue' software

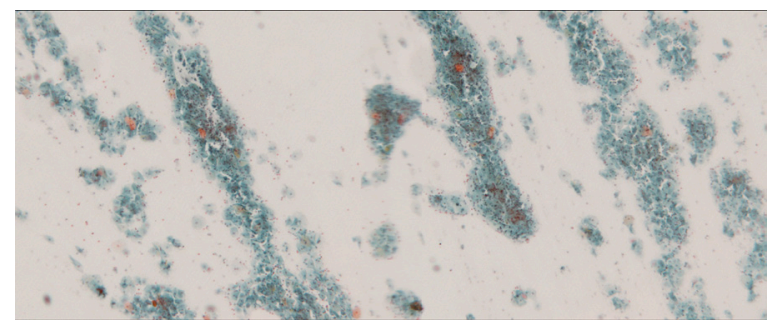

b) Mosaicking result of the two images 'Fig.9' by the developed software

Figure 10.

\section{COMPRESSION WITH THE JP2K NORM:}

\section{A. Transmission with progressive resolution:}

First bits are used to build a part of the picture, as one goes along that bits comes, the resolution (dimension) grows from a factor of two in each direction.

Example: the image has been compressed with three levels of resolution (see Fig. 11).

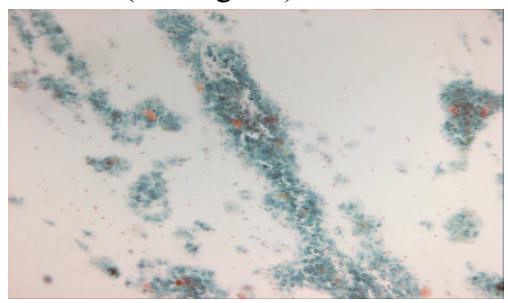

a) Original image

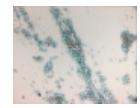

(b) Low resolution picture (resolution 0 ): $6.25 \%$ of the initial image

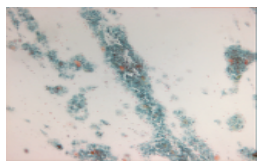

(c) Medium resolution (resolution 1 ): $25,09 \%$ of the initial image

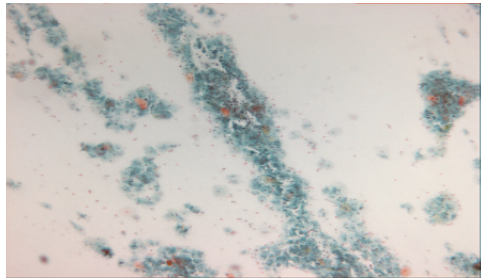

(d) High resolution (resolution 2): 100\% of the initial image

Figure 11. progressivity by layers with three levels of resolution 
This amount to put only blocks from the sub-band LL2 in the Stream-code To obtain such a resolution we add to the sub-band LL2, sub-bands HL2, LH2, and HH2, every blocks code from these sub-bands must be included in the stream-code. Every block codes must figure in the streamcode. The grouping of contributions of block-codes in a same package shape a layer and match to a certain quality of a resolution level and of a component. The definition of several layers in a picture allows to rebuild it at different level of quality.

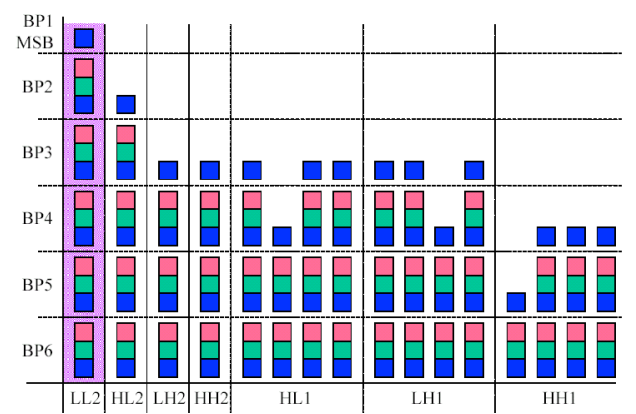

Low resolution, high quality.

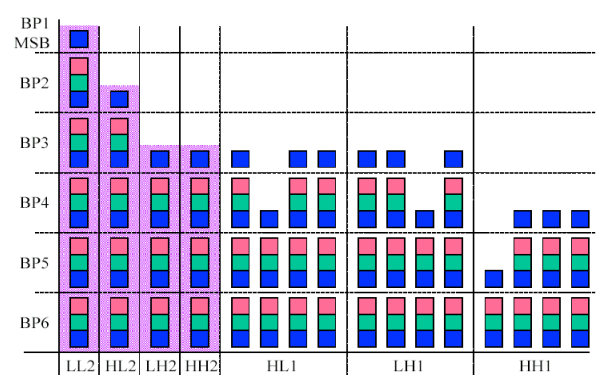

Medium resolution, high quality.

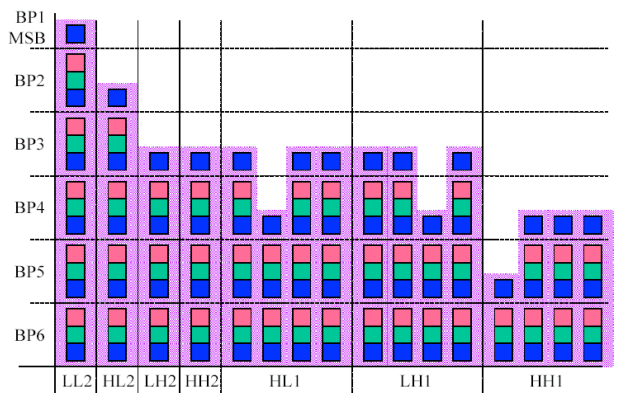

High resolution, high quality

Figure 12. Progressivity by layers

The layer contain the contribution of every block-codes of length $L_{i}$ which minimizes the distortion

$$
D=\sum_{i} D_{i} \text { with } \sum_{i} L_{i} \leq L_{\max } \text {. }
$$

$\mathrm{i}$ : is the block- code number.

D: the distortion brang by each block-code.

When a rate of compression is indicated as an argument of the codec, an intern heuristic determine a lower limit and spaced logarithmically rates of layers in the range.

Example: the original picture (Fig. 11.a) was compressed with twenty layers. To reconstruct the picture in different levels of quality, simply we add each time a layer in a code-stream (See Fig. 13)

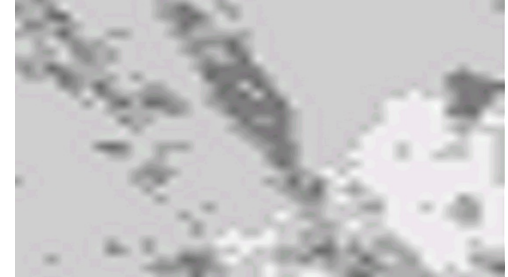

Image with one layer ( layer1 ): $7.36 \%$ from the initial image

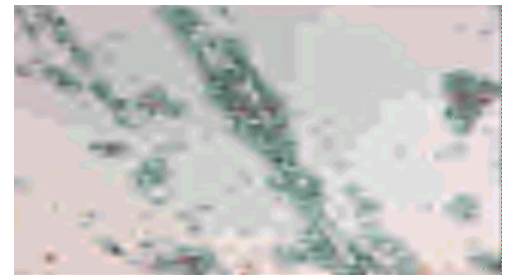

Image with 5 layer ( layers 1,2..,5 ): 9,14\% from the initial image

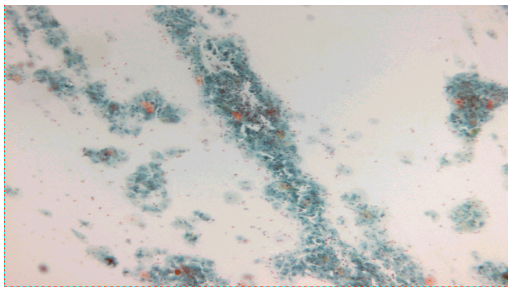

Image with 15 layers ( layers 1,2..,15 ): 16,54\% from the initial image

Figure 13. Example of compression with different layers

\section{B. Progressivity by spatial Region (Spatial Location: Precinct)}

With this type of progression, the image can be received as a database from left to right and from bottom to top.

\section{Progressivity by Component:}

JPEG2000 supports images with 6384 components, this type of progression control decoding order data corresponding to different component, with this type of progression, the image of grey level from a color image will be decoded at first followed by the color information.

JP2K norm defines at all five types of progression by combination of the above methods[12]

1) Layer-resolution-Component-Position Progression (LRCP) [13].

For each layer, for each resolution, for each component, for each position, include the data's packets.

This progression is mainly «by quality», packages of layer 0 appear in the code-stream for each resolution, component and precinct before the packages of layer1, the quality is getting better in the entire image at each index layer's increment.

\section{2) Resolution-Layer-Component-Position} Progression (RLCP)

For each resolution, for each layer, for each component, for each position, include the data's packets.

This progression in mainly «by resolution», packets of the resolution 0 appear in the code-stream in full quality, including all layers in each component and precinct, before the introduction of resolution 1.

3) Resolution-Position-Component-layer Progression (RPCL) 
For each resolution, for each position, for each component, for each layer, include the data's packets.

This progression in mainly «by resolution», the progression inside a data resolution and by position.

4) Position-Component-Resolution-Layer Progression (PCRL)

For each position, for each component, for each resolution, for each layer, include the data's packets.

This progression in mainly «by position», the codestream progresses from top to bottom of the image.

5) Component-Position-Resolution-Layer Progression (CPRL)

For each resolution, for each position, for each component, for each layer, include the data's packets.

This progression is mainly «by component», until all packets of component 0 above all packets of component 1 etc. These different modes are presented in the table IV.

TABLE IV.

DIFFERENT TYPE OF PROGRESSIVITY BY COMPONENT

\begin{tabular}{|c|c|c|c|c|c|}
\hline & LRCP & RLCP & RPCL & PCRL & CPRL \\
\hline $\begin{array}{c}0.703 \\
\text { bpp }\end{array}$ & & & & & \\
\hline $\begin{array}{c}0.999 \\
\text { bpp }\end{array}$ & & & & & \\
\hline
\end{tabular}

VI. RESULTS AND DISCUSSION:

The decoding at the output of the camera allows to improve the efficiency of the telediagnosis chain and to correct the crystallization defaults.
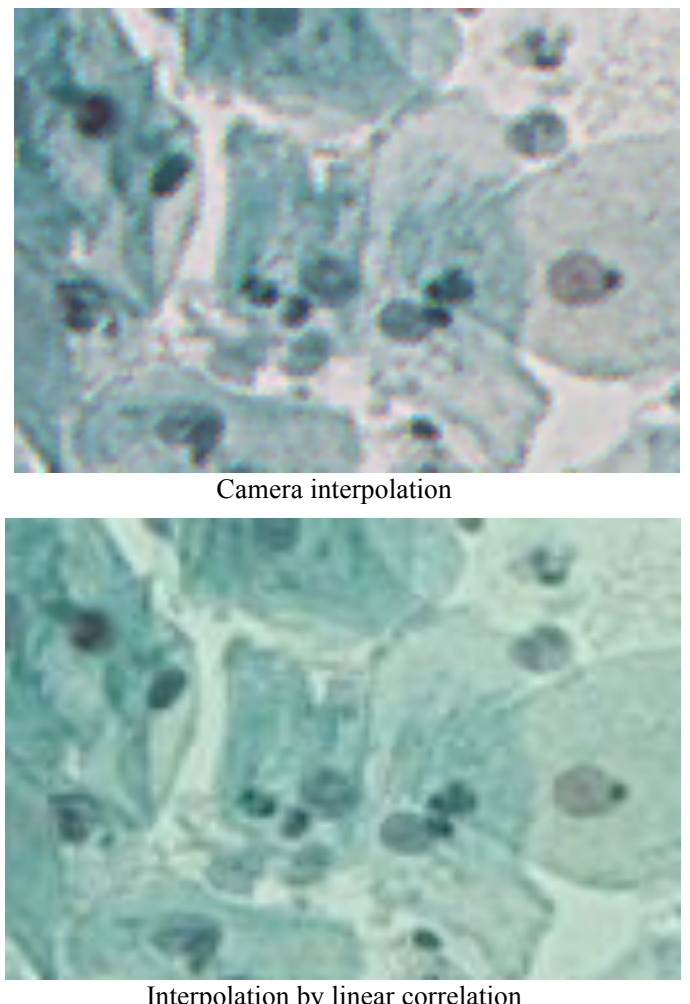

Interpolation by linear correlation
The implemented mosaicking allows us to optimize the computation time and to improve then the efficiency of the telediagnosis chain.

We remind that the photometry correction is very efficient when using the 'shading' images of each elementary image of the slide.
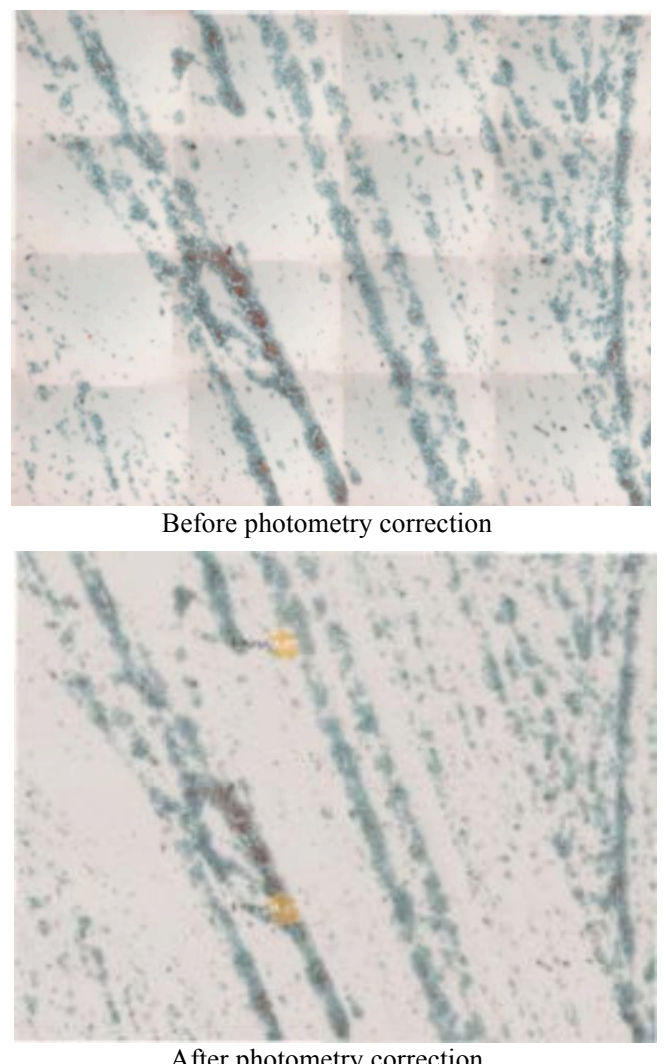

The Table 1 shows the evolution of display with different modes, progressivity in the LRCP mode shows an advantage over the other in this way we approach the quality of the final image faster than other types of progressivity.

\section{CONCLUSION:}

In this article, we have solved the problem of the grid which appears in the mosaicked images.

We have discussed the different processings of the camera issued image before and after interpolation.

The 'Bayer' image processing is very competitive, allowing to reduce the computation time.

The shading correction can be applied on the camera issued images; this speeds up the execution time three times, (division over one channel instead of three channels in the coloured images).

After that, a decoding will be applied on the correction results to obtain the coloured images.

The mosaicking allows the reading of a big size and strong magnification slide in network, in the frame of a diagnosis protocol which develops, among others, a remote consultation system of a big size virtual slide for the medical diagnosis in haematology.

Finally we have study the mechanisms of progressivity offered by JPEG2000, the possibility to see the progressive display of an image and the virtual slide this standard. 


\section{REFERENCES}

[1] Yu-Chieh Chen and Tai-Shan Liao, National Applied Research Laboratories, Hsinchu, Taiwan; Edited by Martin Rowe - Granville - February 4, 2010.

[2] B. E. Bayer "Color imaging array" U.S. patent 3, 971, 065 (1976).

[3] W. Lu, Y. Tan, "Color filter array demosaicking: New method and performance measures", IEEE Trans. on Image proc., vol.11, no. 9 , Oct. 03.

[4] B. K. Gunturk, Y. Altunbasak, R. M. Mersereau, "Color plane interpolation using alternating projections", IEEE Trans. on Image Proc., vol. 11, no. 9, Sept. 02.

[5] J.F. Hamilton Jr., J.E. Adams, "Adaptive color plan interpolation in single sensor color electronic camera", U.S. Patent $5,629,734$ (1997).

[6] Pascal Getreuer, Image Interpolation with Geometric Contour Stencils, Image Processing On Line, 2011

[7] Friedrich M. Wahl, Digital image signal processing, Artech house Boston.

[8] S. Ozeré, P. Bouthemy, F. Spindler, P. Paul-Gilloteaux, C. Kervrann. Robust parametric stabilization of moving cells with intensity correction in light microscopy image sequences. IEEE Int. Symp. on Biomedical Imaging: from nano to macro (ISBI'2013), San Francisco, April 2013.
[9] Système et procédure pour l'authentification d'images. Huawei Technologies 5, avril 2012.

[10] Bacuslab Project availbale on http://www.bacuslabs.com/indexvirtmic.html

[11] Matchslide Project available on http://www.tribvn.com/asp

[12] JPEG2000 Image Transmission. Electronic Design, Test and Applications, 2008. DELTA 2008. Pages :253 - 257

[13] Gabriel Mart'n,a Vicente Gonz'alez-Ruiz,b Antonio Plaza,a Juan P. Ortiz,b.Impact of JPEG2000 compression on endmember extraction. Journal of Applied Remote Sensing, Vol. 4, 041796 (14 July 2010) Department of Technology of Computers and Communications, University of Extremadura.

Waheb LARBI, Allal LARBI, and Bachir DJEDOU are with the Laboratoire d'Etude et de Recherche en Instrumentation et en Communications de Annaba (LERICA), Département d'Electronique, Faculté des Sciences de l'Ingénieur, Université Badji Mokhtar - Annaba, B.P. 12, 23000-Annaba, Algeria (larbi.paper@gmail.com, allal.larbi@univ-annaba.org,_bachir.djedou@univannaba.org).

Submitted 23 March 2014. Published as re-submitted by the authors 12 May 2014. 